\title{
A FACTORIZATION OF STABLE HOMEOMORPHISMS OF $E^{n}$
}

\section{ELLARD NUNNALLY}

Introduction. In [2] it was shown that any stable homeomorphism of the $n$-sphere $S^{n}(n>0)$ could be factored into the product of two topological dilations. This result was obtained by making repeated use of the invertibility properties of the sphere. A similar, though slightly weaker, proposition is true, and is proved in this paper, for the stable group on Euclidean $n$-space, $E^{n}$. However, since $E^{n}$ is not invertible, a procedure somewhat different from that employed in [2] must be followed here.

Definitions and notation. If $n$ is a positive integer, we denote by $E^{n} n$-dimensional Euclidean space, by $G$ the group of stable homeomorphisms of $E^{n}$ onto itself, by $H$ the subgroup of $G$ consisting of all those homeomorphisms of $E^{n}$ onto itself each of which is supported on some $n$-cell, and by $\mathfrak{C}$ we mean the collection of all images under the elements of $H$ of the unit $n$-cell in $E^{n}$. If $D \subset E^{n}$, we denote the interior of $D$ by $D^{0}$. If $f, g \in G$, then $g f g^{-1}$ is called a stable conjugate of $f$.

Definition 1. If $p \in E^{n}$ and $\left\{D_{i}\right\}_{i=-\infty}^{\infty}=D \subset \mathcal{C}$, then the pair $(p, D)$ is called a dilation structure on $E^{n}$, provided

(a) $D_{i} \subset D_{i+1}^{0}, \quad-\infty<i<\infty$,

(b) $\cap D=\{p\}$, and

(c) $U D=E^{n}$.

Definition 2. An element $d$ of $G$ is called a topological dilation on $E^{n}$ if there exists a dilation structure $(p, D)$ on $E^{n}$ such that $d\left(D_{i}\right)$ $=D_{i+1},-\infty<i<\infty$. In this case $(p, D)$ is said to be a dilation structure for $d$, and $d$ is said to be carried by $(p, D)$.

An example of a canonical topological dilation, $d$, is a homeomorphism of $E^{n}$ onto itself which expands radially outward from the origin. That is if $x \in E^{n}$, then $d(x)=r x$ where $r$ is a fixed real number greater than 1 . Here $d$ is carried by the dilation structure $\left(p,\left\{d^{i}(C)\right\}_{i=-\infty}^{\infty}\right)$, where $p$ is the origin and $C$ is the unit $n$-cell centered at the origin. Clearly any stable conjugate of $d$ is a topological dilation, and from Theorem 1 below we may conclude the converse: that any topological dilation is a stable conjugate of $d$.

Presented to the Society, October 20,1966; received by the editors October 12, 1966. 


\section{Two lemmas and a theorem.}

Lemma 1. Suppose $K_{0}, K_{1} \in \mathcal{C}$ with $K_{1} \subset K_{0}^{0}$ and $g \in G$ with $g\left(K_{0}\right)$ $\subset K_{1}^{0}$. Suppose also that $\left\{D_{i}\right\}_{i=1}^{\infty} \subset \mathcal{e}, D_{i+1} \subset D_{i}^{0}, i \geqq 1, D_{1} \subset K_{1}^{0}$, and $\cap\left\{D_{i}\right\}_{i=1}^{\infty}$ is a single point. Then there exists $h \in H$, supported on $K_{1}$ such that $(h g)^{i}\left(K_{0}\right)=D_{i}$, for $i \geqq 1$.

Lemma 2. Any dilation structure carries a topological dilation.

Proof. See the proofs of Lemma 2 and Lemma 3 in [2].

Theorem 1. Suppose $d_{1}$ and $d_{2}$ are topological dilations on $E^{n}$. Then there exists an $f \in G$ such that $d_{1}=f d_{2} f^{-1}$.

Proof. The proof of this follows from Theorem 1 in [2] along with a couple of observations. If $h$ is a stable homeomorphism of $E^{n}$ onto itself then its natural extension to $S^{n}$ obtained by leaving fixed the point at infinity is stable on $S^{n}$; and conversely, if $h$ is stable on $S^{n}$ and leaves the point at infinity fixed, then $h$ restricted to $E^{n}$ is stable on $E^{n}$. Thus, if, in Theorem 1 in [2], one considers $g_{1}$ and $g_{2}$ to be the extensions to $S^{n}$ of $d_{1}$ and $d_{2}$ respectively, then the stable homeomorphism $r$ constructed there does leave the point at infinity fixed; and therefore we may take $f=r \mid E^{n}$.

The Factorization Theorem. Suppose $f \in G$ and $f$ is not the identity. Then there exist topological dilations, $d_{1}$ and $d_{2}$, such that $d_{1} f=d_{2}$.

Proof. Since $f$ is not the identity, there exists a point $p_{1}$ such that $p_{1} \neq f\left(p_{1}\right)=p_{2}$. Select $C_{0}, D_{1} \in \mathcal{e}$ with $p_{1} \in C_{0}^{0}, C_{0} \subset D_{1}^{0} \supset f\left(C_{0}\right)$, and $f\left(C_{0}\right) \cap C_{0}=\varnothing$. We may also select $\left\{D_{i}\right\}_{i=2}^{\infty} \subset$ e with $\bigcup\left\{D_{i}\right\}_{i=1}^{\infty}=E^{n}$ and such that $D_{i} \subset D_{i+1}^{0}$ and $D_{i} \subset f\left(D_{i+1}^{0}\right)$ for $i \geqq 1$.

Now set $D_{0}=f\left(C_{0}\right)$ and select $\left\{D_{i}\right\}_{i=-1}^{-\infty} \subset \mathbb{C}$ with $\cap\left\{D_{i}\right\}_{i=-1}^{-\infty}=\left\{p_{2}\right\}$ and $D_{i} \subset D_{i+1}^{0}$ for $i \leqq-1$. Since $\left(p_{2},\left\{D_{i}\right\}_{i=-\infty}^{\infty}\right)$ is a dilation structure, by Lemma 2 it carries a topological dilation $d$. That is $d\left(D_{i}\right)=D_{i+1}$ for $-\infty<i<\infty$.

Set $g=f^{-1} d^{-2}$ and consider $g\left(D_{1}\right): d^{-2}\left(D_{1}\right)=D_{-1} \subset D_{0}^{0}=f\left(C_{0}\right)$. Therefore $g\left(D_{1}\right)=f^{-1} d^{-2}\left(D_{1}\right)=f^{-1}\left(D_{-1}\right) \subset f^{-1}\left(D_{0}^{0}\right)=C_{0}$. Set $K_{1}=g\left(D_{1}\right)$ and $K_{0}=C_{0}$. Since $C_{0} \subset D_{1}^{0}, g\left(C_{0}\right) \subset g\left(D_{1}^{0}\right)=K_{1}^{0}$. Hence $g\left(K_{0}\right) \subset K_{1}^{0}$. Now select $q \in K_{1}^{0}$ and $\left\{F_{i}\right\}_{i=1}^{\infty} \subset \mathcal{C}$ with $F_{1} \subset K_{1}^{0}, \cap\left\{F_{i}\right\}_{i=1}^{\infty}=\{q\}$, and $F_{i+1} \subset F_{i}^{0}$ for $i \geqq 1$. By Lemma 1 there exists $h \in H$, supported on $K_{1}$, such that $(h g)^{i}\left(K_{0}\right)=F_{i}$ for $i \geqq 1$.

We now show that $r=d^{2} h f h^{-1}$ is a topological dilation carried by $\left(q,\left\{r^{i}\left(C_{0}\right)\right\}_{i=-\infty}^{\infty}\right)$. Consider $r\left(C_{0}\right)$. Since $h$ is supported on $K_{1}$ and $K_{1} \subset C_{0}^{0}, h^{-1}\left(C_{0}\right)=C_{0}$; also $f\left(C_{0}\right)=D_{0}, h\left(D_{0}\right)=D_{0}$, and $d^{2}\left(D_{0}\right)=D_{2}$. Therefore $r\left(C_{0}\right)=D_{2}$. Now we consider $r^{2}\left(C_{0}\right)=r\left(D_{2}\right)$. Since $h$ is supported on $K_{1}, h^{-1}\left(D_{2}\right)=D_{2}$; also $f\left(D_{2}\right) \supset D_{1}$. Thus $h f\left(D_{2}\right)=f\left(D_{2}\right)$. 
Hence $r\left(D_{2}\right)=d^{2} f\left(D_{2}\right) \supset d^{2}\left(D_{1}\right)=D_{3}$. Continuing in this manner it is easy to see that $r^{i}\left(C_{0}\right) \supset D_{i}$ for $i \geqq 1$. From this and the fact that $C_{0} \subset r\left(C_{0}^{0}\right)$ we conclude that $r^{i}\left(C_{0}\right) \subset r^{i+1}\left(C_{0}\right)$ for $-\infty<i<\infty$ and $\cup\left\{r^{i}\left(C_{0}\right)\right\}_{i=-\infty}^{\infty}=E^{n}$. It only remains to show that $\{q\}=\bigcap\left\{r^{i}\left(C_{0}\right)\right\}_{i=0}^{-\infty}$. Consider $r^{-1}\left(C_{0}\right)=h f^{-1} h^{-1} d^{-2}\left(C_{0}\right)$. Since $d^{-2}\left(C_{0}\right) \subset D_{0}, h^{-1} d^{-2}\left(C_{0}\right)$ $=d^{-2}\left(C_{0}\right)$. Hence $r^{-1}\left(C_{0}\right)=h f^{-1} h^{-1} d^{-2}\left(C_{0}\right)=h f^{-1} d^{-2}\left(C_{0}\right)=h g\left(C_{0}\right)=F_{1}$. Now consider $r^{-2}\left(C_{0}\right)=r^{-1}\left(F_{1}\right)$. Again, since $d^{-2}\left(F_{1}\right) \subset D_{0}$, we have that $h^{-1} d^{-2}\left(F_{1}\right)=d^{-2}\left(F_{1}\right)$, and consequently $r^{-1}\left(F_{1}\right)=h f^{-1} h^{-1} d^{-2}\left(F_{1}\right)$ $=h f^{-1} d^{-2}\left(F_{1}\right)=h g\left(F_{1}\right)=F_{2}$. Continuing in this manner we see that $r^{-i}\left(C_{0}\right)=F_{i}$ for $i \geqq 1$. Hence $\{q\}=\bigcap\left\{r^{i}\left(C_{0}\right)\right\}_{i=0}^{-\infty}$ and $r=d^{-2} h f h^{-1}$ is a topological dilation. Observing that $d_{1}=h^{-1} d^{2} h$ and $d_{2}=h^{-1} r h$ are topological dilations and that $d_{1} f=d_{2}$ completes the proof of the theorem.

Corollary. Suppose $d$ is a topological dilation and $f$ is a stable homeomorphism. Then $f$ is the product of a stable conjugate of $d$ by a stable conjugate of $d^{-1}$.

Proof. If $f$ is the identity, then $f=d d^{-1}$. Suppose $f$ is not the identity. Then by Theorem 2 there are topological dilations $d_{1}$ and $d_{2}$ such that $d_{1} f=d_{2}$. By Theorem 1 there exist stable homeomorphism $r_{1}$ and $r_{2}$ such that $d_{1}=r_{1} d r_{1}^{-1}$ and $d_{2}=r_{2} d r_{2}^{-1}$. Hence $r_{1} d r_{1}^{-1} f=r_{2} d r_{2}^{-1}$, and therefore $f=\left(r_{1} d^{-1} r_{1}^{-1}\right)\left(r_{2} d r_{2}^{-1}\right)$.

\section{BIBLIOGRAPHY}

1. Morton Brown and Herman Gluck, Stable structures on manifolds. I, Ann. of Math. 79 (1964), 1-17.

2. Ellard Nunnally, Dilations on invertible spaces, Trans. Amer. Math. Soc. 123 (1966), 437-448.

UNIVERSITY OF MASSACHUSETTS 